\title{
Was haben Assistenzärztinnen und Assistenzärzte zu managen?
}

C. Schmitz, P. Berchtold

Wozu sollten sich Assistenzärztinnen und Assistenzärzte mit Management beschäftigen? Sind nicht gerade sie diejenigen, die am wenigsten zu managen haben? Sind nicht sie diejenigen, die sich in den am wenigsten einflussreichen Positionen in der medizinischen Hierarchie befinden? Sind nicht sie diejenigen, die ausreichend damit beschäftigt sind, ihr medizinisches Fachwissen zu vertiefen? Zweifellos sind diese Fragen sehr berechtigt und gleichwohl lohnt sich für Assistenten die Beschäftigung mit den Grundlagen des Managements. Denn neben ihrer Weiterbildung zum Facharzt und Vorbereitung zu leitenden Funktionen sind sie diejenigen, die die Hauptlast der Arbeit in Spitälern tragen und für die die FMH dazu ein Weiterbildungsangebot finanziert.

\section{Management. Immer mehr, immer öfter}

Die Bedeutung des Managements in Spitälern und anderen Institutionen des Gesundheitssystems ist weiter im Steigen begriffen. Den Hintergrund dafür bilden nicht nur der ökonomische Druck, sondern auch eine Reihe zusätzlicher Fragestellungen wie z.B. zur Qualität oder zur Angemessenheit medizinischen Handelns oder zur Ethik. Diesen Fragestellungen können sich die Medizin und ihre Institutionen nicht entziehen. Die Folge dieser Einflüsse ist ein höherer Steuerungsbedarf dieser Organisationen. Verwaltung - verstanden als die blosse Administration eines gegebenen und nicht $\mathrm{zu}$ hinterfragenden «Geschäftsmodells» - ist angesichts heutiger Steuerungsnotwendigkeiten zu wenig, erforderlich sind Aktivitäten wie Prozessoptimierungen und Strukturgestaltungen, Ressourcenverteilung, Personalpolitiken, Schwerpunktbildung usw. Damit steht Management im klinischen Alltag. Management kommt immer dann ins Spiel, wenn Ressourcen knapp sind und strukturelle Spannungen auftauchen. Ist das der Fall, erscheint der Beelzebub «Management» wie Mephisto dem Faust beim Osterspaziergang und singt wie weiland in den 80er Jahren Geier Sturzflug: «Jajaja, jetzt wird in die Hände gespuckt, wir steigern das Bruttosozialprodukt ...»

\section{Arzt und Management}

Die Karriere des Managements ist im Alltag der Medizin zu spüren, z.B. dann, wenn noch mehr Listen $\mathrm{zu}$ administrieren sind (ohne dass man deren Sinn zweifelsfrei eruieren könnte) und noch mehr Codierungen von Krankheiten vorzunehmen sind, die der Individualität der $\mathrm{Pa}$ tienten wenig entsprechen. Der Bedeutungszuwachs ist aber auch, wenn sich unter den Ärzten Unruhe breitmacht, weil sich «Management» in fachliche Dinge einzumischen scheint, wenn sich das Gefühl breitmacht, dass den klinischen Notwendigkeiten zuwenig Aufmerksamkeit geschenkt wird, wenn Einzelfälle zulasten von Durchschnitten ignoriert werden etc. Kurz, «Managen» ist zunehmend präsent, mal mit positiver, mal mit negativer Ausprägung.

Management ist nicht mehr wegzudenken und wird die Zukunft der Ärzte begleiten. Egal ob diese das begrüssen oder verdammen. Schon darum lohnt es sich zu wissen, womit man es denn beim «Managen» eigentlich zu tun hat. Taucht man kurz in die Thematik ein, dann zeigen sich jenseits der raschen Urteile folgende Sachverhalte:

- Management ist nicht mit Ökonomie gleichzusetzen. Zwar hat Management mit betriebswirtschaftlichen Kalkülen zu tun, aber gleichzeitig und intensiv - und nur das ist sinnvolles Management - damit, den «Eigensinn» einer Organisation hinreichend zur Geltung zu bringen. Das gilt in besonderem Masse für Expertenorganisationen wie Spitäler.

- Management ist nicht gleich Zählen und Messen. Zwar wird gerne vor allem das gesteuert, was gemessen und gezählt werden kann, doch umfasst Managen ebenso die Förderung von Menschen, die Gewinnung von Zielen, die Organisation von Prozessen und Strukturen, das Treffen von Entscheiden, das Verteilen von Ressourcen etc. Die Gestaltungsdimensionen des Managements sind wesentlich vielfältiger (und spannender), als gelegentlich ärztliche (Ablehnungs-)Reaktionen ahnen lassen.

- Management ist nicht etwas, das nur ausserhalb der Profession Medizin passiert. Ärzte 
managen die ganze Zeit: Ihre Kliniken, ihre Patienten, ihren Nachwuchs, ihre Forschung. Assistenten im besonderen managen ihre Stationen, ihre Arbeitsabläufe, ihre Zeit und nicht zu vergessen - sich selbst ...

Es geht also ums Managen, d.h. das Gewahrwerden der relevanten Handlungsspielräume, aber auch Rahmenbedingungen, in denen man sich befindet - den Kontext verstehen -, und das Wissen und Können um die Interventionsmöglichkeiten, die hierin gegeben sind, Aufgaben und Werkzeuge kennen und können: z. B. im Arbeitsalltag der Bettenstationen. Das Medium der Intervention ist immer Kommunikation. Wie sonst könnten wir uns verständigen. Die Kunst der effektiven, wirkungsvollen Kommunikation will (früh im beruflichen Werdegang) gelernt sein, darum muss sich jeder integrative Managementansatz intensiv mit der Kommunikation beschäftigen. Für Experten liegt hier ein Mehrwert darin, dass sie auch in vielen fachlichen Feldern auf effektive und sorgfältige Kommunikation angewiesen sind (gegenüber Patienten, Kollegen, Pflegenden, der Öffentlichkeit).

\section{Lebendiges Managen}

Betrachten wir Managen in unserem integrativen Verständnis, dann sehen wir, dass es um ein lebendiges, facettenreiches Intervenieren in Organisationen geht und dass es ein wesentlich kommunikatives Unterfangen darstellt. Lebendig wird es vor allem dadurch, dass es um Mehrdeutigkeiten und Ungewissheiten geht und dass auf wenig «evidence» zurückzugreifen ist. Klar hat Managen mit vernünftigen Argumenten, systematischen Analysen, rationalen Prozessen etc. zu tun, aber es umfasst viel mehr. Die englische Bedeutung des Begriffs «to manage» lässt uns diese weiträumige Bedeutung nachvollziehen (im Deutschen wird der Begriff eingeschränkter und verengter konnotiert). «Would you manage to drink another whisky?» meint die Frage, ob sich, angesichts der Ungewissheit der Entwicklung der Durststrecke, noch ein Gläschen Malt ausgehen wird oder bereits nicht mehr. Schafft man's dann noch nach Hause? Die Wortbedeutung erstreckt sich also gerade auf die Behandlung mehrdeutiger, ungewisser Situationen - und eben davon handelt Management im Kern, wenn man es recht versteht. Das gilt um so mehr, als sich heute an vielen Ausbildungs- und Weiterbildungsorten und gerade auch in der Medizin ein recht reduziertes und eindimensionales Verständnis verbreitet hat. Das macht Management in Spitälern doppelt interessant.

Also, was haben Assistenzärztinnen und Assistenzärzte zu managen: Could you manage to take another training? 